\title{
LA DOCUMENTACIÓN EN LOS ESTUDIOS DE COMUNICACIÓN: APLICACIÓN DE HERRAMIENTAS 2.0
}

\author{
Maribel Serra-Pallarés y Sílvia Espinosa-Mirabet
}

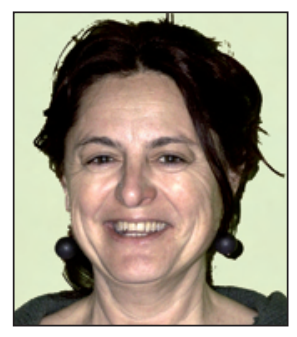
diaris de Pascal (2008) y Anatomia d'un rei (2010).

Maribel Serra-Pallarés es licenciada en geografía e historia por la Univ. Autònoma de Barcelona, master en estudios sobre las mujeres por la Univ. de Barcelona (UB) y postgrado en Patrimonio cultural. Trabaja desde hace 20 años en el Departamento de Documentación de TV3 y ha sido profesora asociada de Documentación audiovisual de la Univ. Rovira i Virgili y de Gestión de la información en la Univ. Ramon Llull. Su especialidad es la documentación aplicada al mundo audiovisual y la realización de trabajos de documentación e investigación para reportajes y documentales. Ha trabajado, entre otros, en 30 minuts; El preu de la memòria (2001); Els nens perduts del franquisme (2002, Premi nacional de periodisme); la serie documental Històries de Catalunya (2003-2005); Els

Dept. de Documentació de TV3, Televisió de Catalunya Carrer de la TV3, s/n. 08970 Sant Joan Despí (Barcelona) mserra.s@tv3.cat

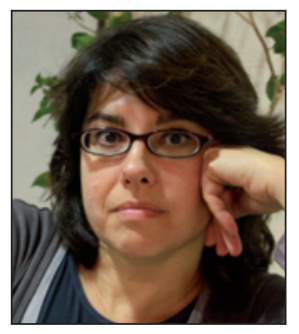

Sílvia Espinosa-Mirabet es doctora en comunicación audiovisual, licenciada en ciencias de la información, master en dirección de comunicación para empresas e instituciones y master en comunicación audiovisual y publicidad, todo ello por la UAB. Sus investigaciones se centran en la historia de los medios audiovisuales desde una perspectiva de género, y la innovación en la docencia universitaria. Trabaja en el documental Locutoras y mujeres: las voces olvidadas de la historia de la radio en Girona. Ha trabajado como periodista en la Cadena SER, en radio y TV local y como corresponsal o colaboradora, entre otros en El país y Diari de Barcelona. Es profesora de radio en la UAB y en la UDG donde dirige el Diploma d'Especialització Integral de Ràdio. Ha sido profesora de Gèneres informatius i audiovisuals II en la Univ. Rovira i Virgili.

Universitat de Girona Fac. de Lletres, Dept. de Filologia i Comunicacio PI. Ferrater Mora, 1. Campus Barri Vell. 17071 Girona silvia.espinosam@udg.edu

\section{Resumen}

Se describe una experiencia llevada a cabo en el tercer curso de los estudios de Comunicación de la Universitat Rovira $i$ Virgili (URV), con el fin de incentivar a los alumnos y cambiar su percepción de la asignatura Documentación informativa. Tradicionalmente esta materia tan importante en las enseñanzas de comunicación no ha sido vista por los estudiantes como una disciplina versátil y útil que ayude a garantizar la calidad en sus trabajos. Por ello, para demostrar su utilidad se preparó un programa de cooperación con otra asignatura del mismo curso: Géneros informativos y audiovisuales. Entre las dos los alumnos realizarían el trabajo final: un reportaje elaborado en distintos formatos: prensa, radio y televisión. La organización de la información y sus fuentes se plasmaba en la creación de blogs que servirían de almacén del material recopilado.

\section{Palabras clave}

Gestión de la información, Documentación informativa, Web 2.0, Blogs, Cooperación interdisciplinar, Aprendizaje, Comunicación audiovisual, Estudios universitarios, Psicología motivacional.

Title: Documentation in communication studies: applying web 2.0 tools

\begin{abstract}
We describe a case of implementation in the Universitat Rovira i Virgili (URV) for a change of mindset in third-year students taking the Informative Documentation class. Traditionally this area, so important in all the teaching of communication, has been a drag on the learning process of students who don't see it as a versatile and useful subject that will guarantee the quality of their work. For this reason we designed a paired course with Informative and Audiovisual Genres, in the same program. Between the two classes, students completed a final project, a report developed for different media: press, radio and TV. The organization of all of the information and its sources came together in the creation of blogs that stored all of the material as it was collected.
\end{abstract}




\section{Keywords}

Information management, Web 2.0, Blogs, Learning, University studies, Audiovisual communication, Interdisclipinary cooperation, Motivational psychology.

Serra-Pallarés, Maribel; Espinosa-Mirabet, Sílvia. "La documentación en los estudios de comunicación: aplicación de herramientas 2.0". El profesional de la información, 2011, marzo-abril, v. 20, n. 2, pp. 228-233.

\section{Contexto}

Los profesionales de la comunicación sabemos que las técnicas de la documentación son básicas entre otras cosas para crear buenas sinergias de comunicación en cualquier ámbito. Por otra parte, los profesores que impartimos materias de comunicación sabemos que entre nuestro público, los estudiantes, la Documentación informativa no goza de excesivas simpatías, a pesar de que la incorporación de las TIC ha facilitado mucho el trabajo. La mayoría de universidades que imparten esta asignatura en estudios de comunicación audiovisual lo hacen con clases magistrales, a pesar de la evolución experimentada por las técnicas documentales. En algunos centros el término Documentación informativa ha sido sustituido por Técnicas de información o Gestión de la información ${ }^{1}$.

Vivimos en un entorno donde el ciudadano, además de consumir, y gracias a la universalización de la tecnología, puede crear información y difundirla casi inmediatamente mediante multitud de redes y plataformas sociales. Nuestros alumnos pueden realizar reportajes caseros y colgarlos fácilmente en la Red, y por ello se plantean si es necesario aprender Documentación informativa para hacer un reportaje. ¿Qué utilidad tiene? La respuesta a nuestro juicio es clara: un reportaje informativo es el resultado final de un proceso, igual que la elaboración de un trabajo científico, que necesariamente debe empezar por documentar ampliamente la hipótesis de trabajo. Un buen trabajo de documentación conduce y mejora el producto. La documentación en el periodismo es la marca que diferencia un producto profesional de otro amateur (Espinosa-Mirabet et al, 2009 a y b).

\section{La documentación en el periodismo es la marca que diferencia un producto profe- sional de otro amateur}

En el curso 2003-2004, en los entonces nuevos estudios de comunicación de la Universitat Rovira i Virgili $(U R V)^{2}$ de Tarragona se inició la experiencia pionera que relatamos en estas páginas. Las profesoras responsables de Documentación informativa y de Géneros informativos, audiovisuales y para prensa decidimos iniciar un proceso de cooperación entre las dos materias. El objetivo era llevar a cabo entre las dos un mismo trabajo práctico, final, ambicioso y completo ${ }^{3}$, que hiciera ver a nuestros alumnos el papel fundamental de la Documentación informativa en el periodismo audiovisual.
Las dos materias eran obligatorias en el currículo académico de los alumnos de tercer curso de la carrera y compartían un objetivo claro, aprender a aprender. Decidimos rediseñar nuestras respectivas asignaturas, que se habían iniciado en 2001, para, aplicando las directrices del Espacio Europeo de Educación Superior (EEES), comenzar un trabajo de cooperación con la finalidad de que nuestros estudiantes se sintieran mejor preparados. Intentamos diseñar estas asignaturas de forma que les ayudaran a entender su relación de aprendizaje con Documentación informativa para conseguir un cambio de mentalidad y una excelencia en su trabajo final. Llevábamos 3 años de reportajes mediocres.

Los buenos resultados invitan a una reflexión abierta sobre la documentación como competencia transversal de cualquier área académica o profesional

Según la psicóloga de la UDG Ariadna Lleonart (2009), es necesario tener una concepción interaccionista para conseguir aprender con motivación. Los factores psicológicos de los alumnos: cognitivos y afectivos, psicosociales, contextuales y relacionales, tienen que mezclarse adecuadamente si se busca el sentido del aprendizaje, que era lo que nosotras intentábamos encontrar. Queríamos inculcarlo en nuestros estudiantes y para ello contábamos con las herramientas 2.0 que la tecnología de la información ponía a nuestro alcance y que a nuestros estudiantes embelesaban más que a nosotras. Nuestro cambió mental para con ellos implicaría además trabajar sobre su capacidad crítica, elemento tan necesario en el entorno comunicativo actual.

En otras facultades de comunicación Géneros informativos II no mezcla en una misma materia las disciplinas escritas con las audiovisuales ${ }^{4}$. Sin embargo hemos comprobado que esta mezcla enriquece a nuestros estudiantes y les otorga un barniz de comunicadores multimedia partiendo de las bases del periodismo clásico. Por eso también necesitábamos que la documentación fuese la columna vertebral de sus trabajos. Por su parte, Documentación informativa se reestructuró totalmente para primar la experimentación por encima de las exposiciones magistrales que hasta ese momento la caracterizaban.

Ambas asignaturas compartían la evaluación continuada y tutorías, con los objetivos de guiar el proceso, enriquecer la calidad del trabajo práctico e incrementar la motivación del alumnado. Los buenos resultados obtenidos en los trabajos 


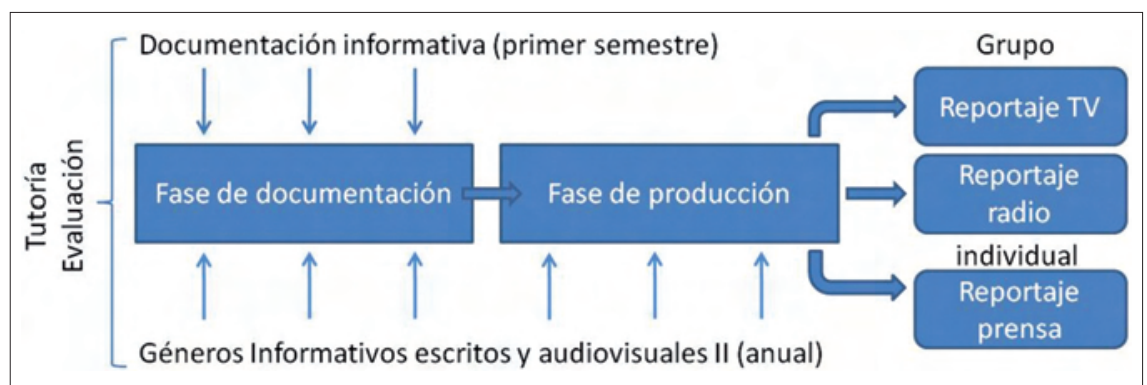

Figura 1. Mapa del diseño de las dos asignaturas ${ }^{6}$

de los alumnos proponen una reflexión abierta sobre la utilización de la documentación como competencia transversal de cualquier área académica o profesional.

\section{Objetivos}

El alumno terminaría dominando las técnicas de búsqueda, organización y análisis de la información para saber escoger las fuentes de información adecuadas y modelar el reportaje, práctica final y más ambiciosa ${ }^{5}$ de Géneros II. Los reportajes tendrían además una difusión más allá del aula mediante el campus virtual Moodle, y mediante su proyección en la televisión local.

Nuestro diseño de cooperación debía asumir además de las competencias ya citadas, el dominio de:

a) códigos y lenguajes: pautas narrativas para expresarse y criterios para analizar los mensajes;

b) herramientas y canales de transmisión de contenidos;

c) sistemas de producción de cada género: adquisición de la capacidad crítica y analítica para la creación de mensajes;

d) sistemas de gestión de la información: adquisición de criterios de selección y verificación de fuentes, y organización de la documentación.

El dominio de los sistemas de gestión de la información necesitaba una herramienta que nos ayudara a conectar con los alumnos para que ellos se sumaran a nuestra propuesta. Si no lográbamos que ellos creyeran en la necesidad de que asumieran esta competencia básica, no lograríamos que aprendieran y dominaran el resto. La herramienta escogida por su versatilidad, facilidad de uso y utilidad fue el blog: un entorno 2.0 en el que todos los miembros del grupo podían trabajar y las profesoras ver y redirigir el trabajo.

\section{Metodología}

Ambas asignaturas eran troncales y por tanto obligatorias. Habitualmente se matriculaban entre 80 y 100 alumnos que dividíamos en 15 grupos de 5 ó 6 alumnos que trabajaban conjuntamente en ambas.

Planteamos un programa para trabajar la etapa de documentación previa al guión y producción del reportaje en Documentación informativa, mostrando así a los alumnos que esta materia -a priori menos agradable para ellos que Géneros II- establece los ejes básicos para un reportaje bien construido, veraz, informativo e interesante. En esta etapa se van generando todas o casi todas las preguntas y dudas referentes al tema del reportaje, su exposición y su fidelidad a la realidad. La búsqueda, organización y recuperación de la información ayuda a ir resolviendo los problemas. Tener un objetivo concreto que genera necesidades auténticas hace que la documentación deje de ser algo esencialmente teórico para convertirse en una solución práctica y útil.

El primer paso fue establecer una hipótesis temática y unos objetivos que determinaran qué había que buscar, qué información podía ser útil. En esta etapa se desarrollan las habilidades lógicas y el método científico de deducción, junto con la explotación de su bagaje intelectual. A menudo la información que se encuentre no sólo condicionará el contenido, sino probablemente también su presentación formal. Esta tarea servirá de base para redactar el guión, elegir entrevistados, redirigir la búsqueda, ilustrar con materiales audiovisuales, gráficos, escoger localizaciones, etc.

\section{Tener un objetivo concreto hace que la documentación se vea como una solu- ción práctica y útil}

Paralelamente a la realización del trabajo científico se diseña el blog. En él se estructura y organiza la información obtenida. Es una buena herramienta de consulta a la que acudir para planificar cada nuevo paso del reportaje: localizaciones, background para entrevistas, contactos o información general. Además exige un esfuerzo suplementario en la organización lógica y en el trabajo en equipo. A estas alturas la tarea realizada en Documentación informativa confirma a los estudiantes su carácter de asignatura principal.

Empieza la segunda etapa: los trabajos se presentan para una primera revisión colectiva. La profesora de Géneros II corrige y orienta dando directrices para que mejoren, cambien o reenfoquen aspectos poco satisfactorios. El hecho de que todos los grupos vean los trabajos del resto de los compañeros sirve también para que cada alumno pueda valorar su trabajo con relación al de los demás: se crea una sana competencia para favorecer una mayor implicación en el sprint final que supone la presentación de los reportajes definitivos dos semanas más tarde. Los alumnos aprenden que gracias al blog, recuperar o incluso encontrar una fuente más adecuada es una tarea más rápida y eficiente.

Documentación informativa, como asignatura semestral, está tocando a su fin y los alumnos deben dejar materiales, fuentes, documentos y datos preparados para emprender la recta final de curso. Ahora trabajar con documentación y fuentes ya no representa ningún desagrado para los estudiantes. Se ha producido un cambio de mentalidad, los blogs de documentación contribuyen a hacer su tarea más sencilla.

En marzo Géneros // retoma el control del reportaje final. Después de revisar los blogs se lleva cabo una tutoría por grupo para planificar enfoques y salidas de campo y así em- 


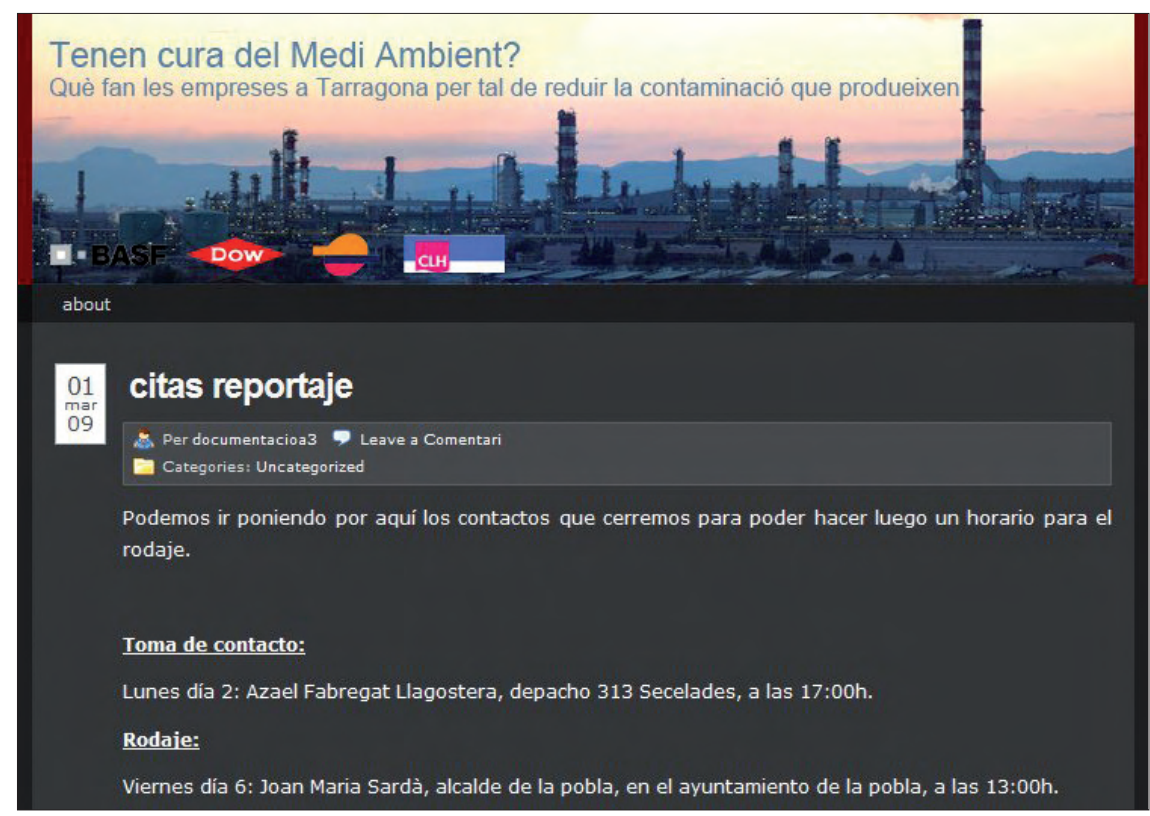

Figura 2. Blog del documental “¿Cuidamos el medio ambiente?” http://documentacioa3.wordpress.com

grande. A raíz de esta experiencia conjunta observamos cómo la asignatura Documentación informativa dejó de ser una materia poco o mal considerada y se convirtió en una puerta de acceso a la aplicación de la web 2.0, valorando la utilidad de las TICs en el contexto profesional de la comunicación.

\section{Resultados}

Al finalizar el primer curso de colaboración anotamos unos resultados sorprendentes. Los grupos de trabajo que habían seguido las directrices marcadas por Documentación informativa en el proceso de documentación de sus reportajes lograban de forma satisfactoria los objetivos planteados. Habían aprendido y además sabían y valoraban que entre los pasos a seguir para

pezar a tejer el reportaje. Empieza el proceso de grabación según una planificación que los estudiantes conocen desde el primer día de curso.

Tras un primer montaje, hay una primera corrección con el objetivo de reorientar, buscar o añadir aspectos poco trabajados. Los alumnos se dan cuenta de la utilidad de su blog y de lo fácil que resulta la tarea de corregir o reorientar cuando la búsqueda de información se hizo adecuadamente. Sólo hay que reconducir aspectos poco claros, supervisar cuestionarios de entrevistas y animar a los alumnos a buscar nuevas vías para contar lo que quieren exponer en su reportaje.

Hay que destacar que en cada corrección los alumnos deben entregar una ficha de reflexión sobre el trabajo llevado a cabo. Las fichas permiten detectar cuáles son las tareas que realiza cada alumno dentro del grupo, observar las sinergias que se establecen y obligar a la reflexión sobre cómo hacen lo que hacen, qué problemas tienen, cómo los han resuelto y qué les ha aportado el trabajo de realización del reportaje ${ }^{7}$.

Se realiza un segundo montaje con post-producción final y se deja la pieza vista para la corrección definitiva. Los alumnos han llegado al final de curso con un reportaje realizado en los lenguajes propios de la televisión, la radio y la prensa escrita.

Después de la corrección final en mayo, se valora el proceso de trabajo llevado a cabo, reflejado en la sucesión de fichas. Se rellena una encuesta -el resultado de la cual adjuntamos en este artículo- y se estudia la posibilidad de colgar en la web de la Facultad algunos de los reportajes. Es también el momento de ver la viabilidad de presentar estas producciones a distintas convocatorias de premios o de "venderlo" con permiso de autores y fuentes implicadas a alguna de las televisiones locales de la provincia de Tarragona.

El hecho de que los alumnos sepan que su reportaje puede merecer estos premios hace que se tomen su trabajo muy en serio y que la motivación para cursar Géneros // sea muy conseguir un buen trabajo era crucial la organización de la información. En cambio los equipos de trabajo que hacían la práctica documental de un tema y que después por cuestiones diversas no lo podían abordar (tema poco atractivo o que implicaba rodajes difíciles o en el extranjero) presentaban trabajos muy superficiales, muy lejos de la marca de un reportaje periodístico. A partir de ahí decidimos prohibir los cambios de tema y reforzamos la primera tutoría.

Para evitar en cursos posteriores que hubiera estas desviaciones, la nota que los grupos de trabajo alcanzaban en Documentación les suponía una puntuación extra en la evaluación continuada de Géneros II, en función de unos porcentajes pactados entre ambas asignaturas. La nota de Documentación se conocía en diciembre y la de Géneros // en

\section{El blog motiva al aprendizaje y sirve de almacén de consulta al que acudir para planificar cada nuevo paso del reportaje}

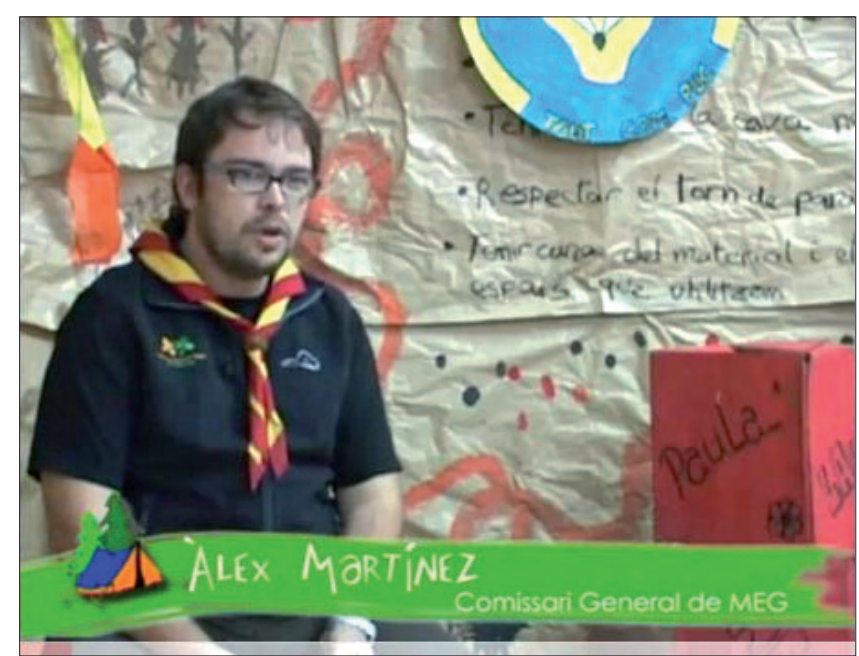

Figura 3. Imagen del documental "Escuela de vida. El escultismo en Catalunya", http://www.youtube.com/watch?v=bGNLPRY4nCc 


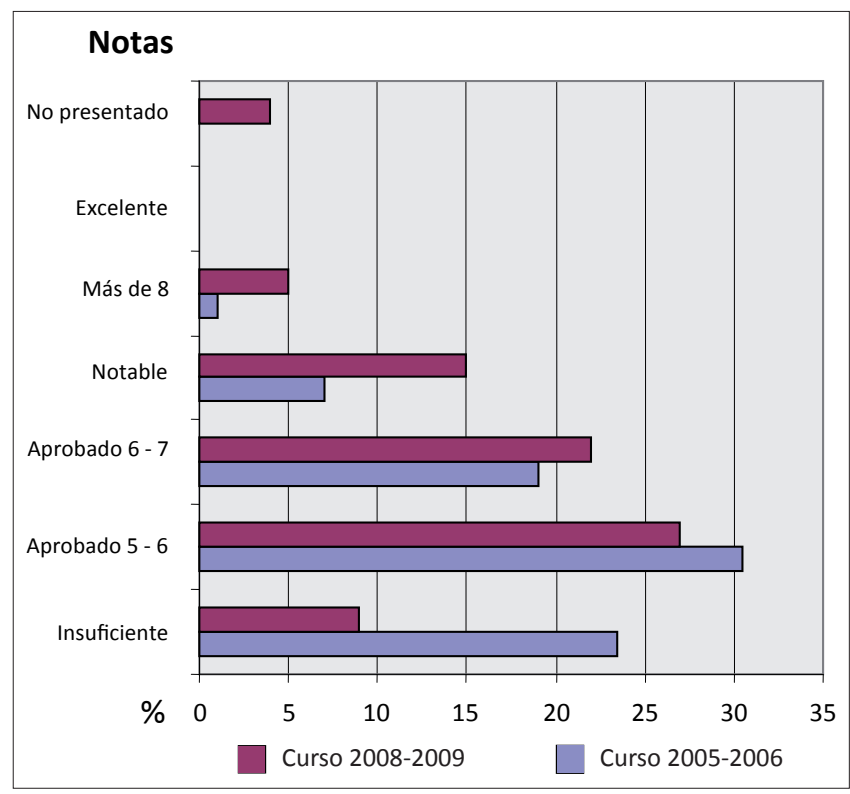

Figura 4. Evolución de las calificaciones finales

junio. Si en la primera se obtenía una puntuación de 7 o más puntos, en Géneros // se sumaba un punto a la nota final.

El reportaje final de Géneros // es un resultado tangible que permite a los alumnos evaluar su propio trabajo y dotarlo de sentido. Incluso si la nota no es lo bastante satisfactoria, la experiencia de aprendizaje resulta enriquecedora; los mismos estudiantes sacan conclusiones sobre la evolución de su trabajo, como veremos más adelante. El trabajo conjunto entre ambas asignaturas implicaba que los alumnos tenían que hacer esfuerzos importantes desde el principio para construir un grupo de trabajo que pasara del aprendizaje cooperativo al colaborativo, según las acepciones de Bermejo (2003). Es decir, tenían que pasar de hacer un reportaje entre todos a hacer uno colectivo que fuera mejor de lo que habrían sido capaces de hacer individualmente y en el que cada miembro del grupo aportara sus conocimientos y habilidades específicas.

Los resultados de las encuestas anónimas realizadas al final del curso 2009 entre todos los alumnos muestran la satisfacción por el trabajo y la seguridad alcanzada con su realización. Hay que resaltar que en el resultado global de la asignatura detectamos que algunos grupos de alumnos fueron mejores realizando sus blogs que su reportaje, cosa que no nos habíamos planteado que pudiese ocurrir al diseñar la colaboración entre las dos asignaturas. En el caso de que esta cooperación se volviese a aplicar habría que solucionar este aspecto. No pretendíamos hacer buenísimos bloggers sino buenísimos periodistas y en este sentido fuimos víctimas de la tecnología, aceptando, que un buen periodista puede ser un excelente blogger pero que a la inversa no es necesario que suceda.

Se comprobó que la mayoría de los estudiantes de las dos asignaturas valoraban la experiencia de realización del reportaje como muy importante y con un alto grado de satisfacción y sólo una minoría de los encuestados indicaron no estar contentos con el reportaje que realizaron.

En el aspecto evaluativo hemos comprobado que las notas finales de nuestros alumnos eran mejores a partir de la cola- boración entre las dos asignaturas. Puede observarse cómo desde 2005 a 2009 disminuyeron los suspensos y aumentaron las notas altas, dato que interpretamos como un mejor desarrollo de las diferentes habilidades y en consecuencia de su grado de motivación por el trabajo cooperativo.

En cuanto a los reportajes concretos, algunos de ellos han merecido menciones o premios:

- Escola de vida. L'escoltisme a Catalunya ganó el V Premi Diputació de Tarragona a la Creativitat Audiovisual $(2009)^{8}$.

- El plan SEQTA: la respuesta al riesgo químico ha ganado en dos ocasiones el accésit al Premi Diputació de Tarragona a la creativitat audiovisual (2008);

- los alumnos del Taller de producción audiovisual, bajo la dirección del profesor de la URV Antoni Pérez-Portabella, han alcanzado el segundo premio del Festival Universitario de Creación Audiovisual Proyecta 08, en el que participaron 20 universidades europeas, y fueron finalistas del festival europeo Memorimage 08.

Las tareas de documentación se enfocaron a todos niveles: creación de guión, contenido de entrevistas, búsqueda de contactos, música e imágenes

\section{Conclusiones}

1. La creación del blog fue el revulsivo que necesitó la materia para que los alumnos integraran la asignatura en su aprendizaje de forma activa y valorasen la gestión de la información como la base indispensable para todo buen trabajo periodístico.

Pueden consultarse los siguientes blogs a modo de ejemplo:

\section{http://www.vegueriesreport.wordpress.com} http://grupc2.wordpress.com http://reciclatgealesescolesdelcamp.wordpress.com http://documentacioa3.wordpress.com http://arqueologiaiurbanisme.wordpress.com

2. Ejercitando las competencias transversales referidas a la búsqueda y la organización de la información ligadas a prácticas de producción real (reportaje) se consigue incrementar la calidad de los contenidos. La tarea de documentación se enfoca a todos niveles: creación de guión, contenido de entrevistas, búsqueda de contactos, música e imágenes. Los alumnos son más responsables de su trabajo y de su proceso de aprender (Benito-Capa; Cruz-Chust, 2007). Observan cómo sólo depende de ellos alcanzar mejor o peor las competencias que ambas asignaturas plantean. El feed-back entre profesores y alumnos aumenta, la relación es más fluida y piden tutorías siempre que les hace falta (incluso en la Red). El profesor adquiere un rol de facilitador.

3. Adquirir estrategias mentales en la búsqueda y la deducción fortalece el trabajo en equipo y su autoestima como grupo. Los valores que implícitamente se aprenden en el trabajo en equipo quedan muy reforzados y sitúan al alumno ante un aprendizaje que tendrá que utilizar en su vida 
profesional fuera de la universidad. La experiencia casi profesional adquirida en este aprendizaje dotó de la seguridad necesaria a una de nuestras alumnas que fuera del ámbito académico se atrevió con el documental La región de Ixán en Guatemala obteniendo el galardón del concurso de Periodismo Solidario Visiones del Sur, convocado por la Coordinadora de ONGs de Euskadi.

Una de las claves del éxito de la experiencia radica en el hecho que nuestros alumnos cursaban la asignatura de Documentación en tercero, cuando ya tenían cierta experiencia en el entorno de la comunicación. Todas las universidades consultadas impartían esta asignatura en primero o segundo: UPF (Barcelona), URL (Barcelona), UC3M (Madrid), UV (Valencia), US (Sevilla), UB (Barcelona), UCM (Madrid), UAB (Barcelona) ${ }^{9}$.

4. Estructurar las asignaturas alrededor de las prácticas y no de la teoría facilita la motivación de los alumnos. Trabajan más y sin reproches aunque les suponga una carga importante de trabajo puesto que encuentran que el aprendizaje es mejor y que les "sirve para algo" concreto. Eso quiere decir que es más importante el hacer y el aprender cómo se hace mientras se hace, que no la definición teórica del género o sus subdivisiones.

5. La universalización tecnológica permite generar escenarios de prácticas muy similares a los del mundo profesional, dándoles seguridad y formación imprescindible para su salida a la "vida real". La democratización de la tecnología permite a los alumnos empezar a convertirse en comunicadores o reporteros de verdad. ¿Qué puede estimular más que eso en alguien que ya tiene una vocación o una tendencia hacia este oficio? Así se da un valor real al trabajo teórico.

Es importante hacer entender a los alumnos que el resultado técnico del reportaje no es un objetivo a perseguir en la asignatura de Géneros II, que evidentemente busca productos que tengan resultados profesionales; sino que pretendemos que sean buenos narradores de historias multimedia y que entiendan que sin contenido no hay ni imágenes, ni sonidos que valgan cuando hablamos de información.

6. Las tutorías son una pieza clave en este tipo de aprendizaje. Son básicas para el buen funcionamiento de las dos asignaturas. Los alumnos tienen unas tutorías de obligado cumplimiento marcadas en el calendario de la asignatura, que pueden hacer tanto virtual como presencialmente.

\section{Notas}

1. Es el caso de la Universitat Ramon Llull (URL) de Barcelona y la Universidad Carlos III de Madrid.

2. En el momento de redactar este artículo las autoras ya no forman parte del equipo de profesores de la URV, aunque la experiencia relatada se llevó a término mientras ambas trabajaban cooperativamente en dicha universidad.

3. Un reportaje informativo sobre un mismo tema que debía realizarse en tres códigos diferentes: radio, televisión y prensa. En el último caso los alumnos debían trabajar de forma individual, en los dos primeros en grupos cooperativos.

4. Éste es el caso de la $U A B$, por poner otro ejemplo más.

5. La realización de tres reportajes sobre el mismo tema pero en distinto formato y con duraciones de 5 y 10 min era una práctica difícil para los alumnos que debían organizarse mucho, trabajar en grupo e individualmente (prensa) y ceder muchas de sus horas, fuera de las aulas, para poder conseguir este fin.

6. Este esquema fue diseñado por Antoni Pérez-Portabella y forma parte de una comunicación presentada en el I Congreso de docencia universitaria de Vigo, 2009.

7. Los referentes audiovisuales profesionales que tienen los actuales estudiantes de comunicación son, desgraciadamente y con demasiada frecuencia, formatos que a simple vista parecen géneros periodísticos en su forma pero que su contenido está llenos de suposiciones, rumores, falsas informaciones o exclusivas que no lo son. Este contenido basura es el que nos obliga a trabajar de una forma estricta y cuidadosa para que estos alumnos que deben ser versátiles con los soportes y tecnológicamente capaces, sean sobre todo periodistas.

8. Este reportaje se puede ver en Youtube: http://www.youtube.com/watch?v=bGNLPRY4nCc

9. Con los estudios de grado, Documentación informativa o Gestión de la información, asignatura ligada a la búsqueda y veracidad de contenidos, ha pasado a ser optativa en la $U C M$, la UAB y la URV. Es una decisión cuanto menos cuestionable.

\section{Bibliografía}

Benito-Capa, Águeda; Cruz-Chust, Ana. Nuevas claves para la docencia universitaria en el Espacio Europeo de Educación Superior. Madrid: Narcea, 2007. ISBN 9788427715011.

Bermejo, Sergio. "Aprendizaje basado en proyectos robóticos". XI Cieet'03 - XI Congreso universitario de innovación educativa en las enseñanzas técnicas. Vilanova i la Geltrú, Barcelona, 2003.

Espinosa-Mirabet, Sílvia; Pérez-Portabella, Antoni; SerraPallarés, Maribel. "La documentación como valor emergente y transversal del aprendizaje". En: Il Jornadas de innovación docente aplicada a las tecnologías de la información y las comunicaciones. Universidad de Alcalá de Henares, 2009.

Espinosa-Mirabet, Sílvia; Pérez-Portabella, Antoni; SerraPallarés, Maribel. "La gestión de la información como competencia clave del aprendizaje". En: Colección formación e innovación universitarias, Portal de formación e innovación educativa na Universidade de Vigo. Vigo: Andavira, 2009, pp. 595-600.

http://webs.uvigo.es/portalvicfie

Lleonart, Ariadna. "Els components individuals i relacionals de l'aprenentatge". Postgrau interuniversitari de docència universitària. Girona: ICE, 2009. 